\title{
A compact low-cost electronic hardware design for actuating soft robots
}

Article

Accepted Version

Oguntosin, V. W., Nasuto, S. J. and Hayashi, Y. (2015) A compact low-cost electronic hardware design for actuating soft robots. International Journal of Simulation Systems, Science and Technology, 16 (3). 6.1. ISSN 1473-8031 Available at https://centaur.reading.ac.uk/40672/

It is advisable to refer to the publisher's version if you intend to cite from the work. See Guidance on citing.

Published version at: http://ijssst.info/Vol-16/No-3/cover-16-3.htm

Publisher: United Kingdom Simulation Society

All outputs in CentAUR are protected by Intellectual Property Rights law, including copyright law. Copyright and IPR is retained by the creators or other copyright holders. Terms and conditions for use of this material are defined in the End User Agreement.

\section{www.reading.ac.uk/centaur}

\section{CentAUR}

Central Archive at the University of Reading

Reading's research outputs online 


\section{A Compact Low-Cost Electronic Hardware Design for Actuating Soft Robots}

\author{
Victoria Wumi Oguntosin \\ Brain Embodiment Lab, \\ School of Systems Engineering \\ University of Reading, \\ Reading, United Kingdom \\ v.w.oguntosin@pgr.reading.ac.uk
}

\author{
Slawomir Jaroslaw Nasuto \\ Brain Embodiment Lab, \\ School of Systems Engineering \\ University of Reading, \\ Reading, United Kingdom \\ s.j.nasuto@reading.ac.uk
}

\author{
Yoshikatsu Hayashi \\ Brain Embodiment Lab, \\ School of Systems Engineering \\ University of Reading, \\ Reading, United Kingdom \\ y.hayashi@reading.ac.uk
}

\begin{abstract}
A low cost, compact embedded design approach for actuating soft robots is presented. The complete fabrication procedure and mode of operation was demonstrated, and the performance of the complete system was also demonstrated by building a microcontroller based hardware system which was used to actuate a soft robot for bending motion. The actuation system including the electronic circuit board and actuation components was embedded in a 3D-printed casing to ensure a compact approach for actuating soft robots. Results show the viability of the system in actuating and controlling siliconebased soft robots to achieve bending motions. Qualitative measurements of uniaxial tensile test, bending distance and pressure were obtained. This electronic design is easy to reproduce and integrate into any specified soft robotic device requiring pneumatic actuation.
\end{abstract}

Keywords-soft robots; electronic circuit; PCB; microcontroller;

\section{INTRODUCTION}

Soft robotics is currently an active area of research [1]-[5] as soft actuators are relatively inexpensive to manufacture. The compliant structure of soft robots allows novel motion and grasping tasks that are difficult to implement with rigid parts [6]. Their compliant nature allows them to be placed in direct contact with the surface of the skin [7] to provide the necessary assistive force through pressure control. These robots are considered soft due to their intrinsic property: the materials from which they are made are compliant and resilient. Soft robots are actuated by only compressed air, they are capable of producing many degrees of freedom for a single actuation source and so are under-actuated. Therefore, pressure control becomes very important when actuating these robots. In order to provide a compact embedded system for pressure control of soft robots, a low cost pressure regulator for robotic application was designed. In this work, an integrated electronic control system and pulse width modulated solenoid valve controls the output pressure proportionally to an internal feedback through an integrated pressure sensor.

The effectiveness of pneumatic pressure controllers has been confirmed through step response experiments, both for PWM-controlled on/off valves [8] as well as for different types of servo valves [9], [10]. Previous work on methods of actuating soft actuators is majorly with the use on/off solenoid valves to control the flow of pressurized air to the actuators [1], [2]. Nonetheless, a complete electronic hardware system for the actuation of soft robots has not been demonstrated so far.

This paper reports the design of a MCU controlled and operated pressure regulator system to perform actuation of soft robots. In order to achieve this low cost system, inexpensive electronic components and 3D printing technology was used. This system offers flexibility since it can be integrated with computer vision system and modified to receive a digital control or analogue signal as input. A computer program written in $\mathrm{C}$ language was implemented for valve control in order to produce an output signal that corresponds with a desired output pressure. Also, tensile strength test was performed on the silicone rubber used to produce the soft actuator in order to determine its tensile properties and fit an hyperelastic material model to describe its behaviour.

The main contribution of this paper is to present a compact, low cost, easy to manufacture hardware system and associated control circuitry for the actuation of pneumatic soft robots. The pressure regulator system consists of a 6VDC air pump, solenoid valves, the MCU control system built on a PCB and a 3D printed case which embeds the whole system to make it low cost, compact and easy to redesign and implement. The ease of production and low cost provides a suitable alternative to expensive off-the-shell electro-pneumatic regulators in the market.

\section{SYSTEM OVERVIEW}

The main aim of this system is to regulate the pressure of compressed air from a pressure source using a low cost and compact electronic circuit such that the output pressure is directed to actuate soft robots. The use of an electropneumatic regulator for this purpose would be expensive. Furthermore, the use of mechanical systems such as cylinders, piston pump would be cumbersome and make the system bulky. Therefore, it is necessary to design a compact electronic system for use in soft robotics.

The system is composed by two main parts, the embedded control on a Printed Circuit board, and the design of a 


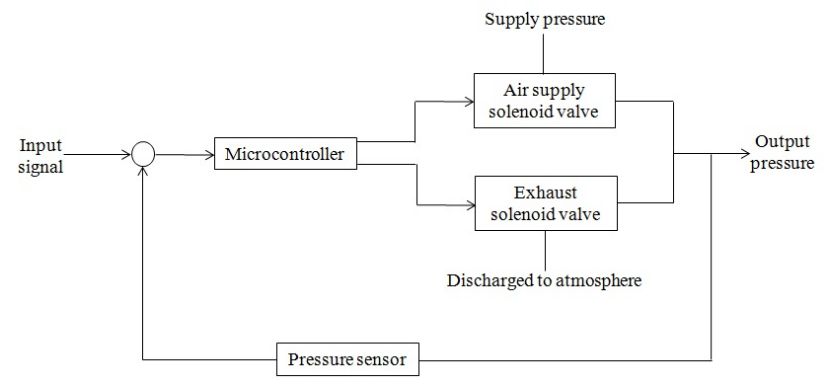

Figure 1. Block diagram of soft actuator pressure controller

compact structure to house the complete pneumatic system so that portability is ensured. Figure 1 shows the block diagram of the proposed application. The input signal is used to set the desired pressure. The realization is by an 8-bit microcontroller (MCU), a PIC16F876A of Microchip running at a clock speed of $4 \mathrm{MHz}$. The MCU system is able to actuate a soft module by reading pressure values via the analogue inputs of the microcontroller. The solenoid valves are controlled by 2 output compare modules configured for PWM-mode. As no operating system kernel is used, mainly interrupt sub-routines and parallel running subunits are used. This works very well for analogue input of pressure signals.

For control of soft actuators by an MCU, 1-way/2-position normally closed solenoid valves was used, ( 2 valves are required for one actuator) one for inflation (inlet solenoid valve) and the other for deflation (exhaust solenoid valve). Solenoid valves control the flow of air into and out of the actuators. Furthermore, $6 \mathrm{~V}$ solenoid valve having a power rating of $2.25 \mathrm{~W}$ solenoid valve, a maximum operating pressure of $350 \mathrm{mmHg}$; exhaust speed of $4 \mathrm{~s}$ and leakage of $3 \mathrm{mmHg} /$ minute was used. These solenoid valves are light and compact enough to be integrated as demonstrated in this work. For higher pressure system, higher pressure valves are needed. This can be achieved by either operating two or more solenoid valves in parallel as one logical valve or simply using valves with higher pressure.

Pressure control is used to control the motion of a soft actuator. To measure pressure, amplified pressure sensors ( 0 - 5psi pressure range) with analogue interface was used having a $1 \mathrm{~ms}$ response time and a 10 bit ADC resolution was used to read the pressure. Using the MCUs PWM features, pressure is controlled by varying the length of the duty cycle. In this case a duty cycle has a maximum length of $1 \mathrm{~ms}$. The output pressure is also dependent on the type of valve, power supply voltage, and pressure difference. This means a specification for the inlet and exhaust solenoid valve (inflation and deflation) is needed. For the inlet solenoid valve, the pressure difference is given by the constant supply pressure and the actual pressure in the actuator. For the exhaust solenoid valve, the pressure difference is between internal pressure and the atmospheric pressure.

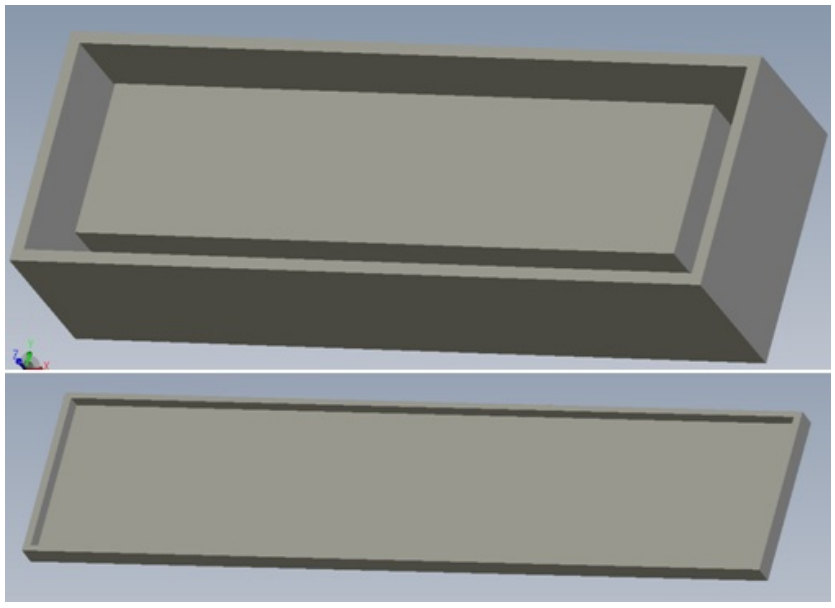

Figure 2. Top and Bottom moulds for a soft robot designed in SolidWorks

Strain energy potential is used to relate stresses to strains in hyperelastic materials rather than using Young's modulus and Poisson's ratio [11]. The stress-strain relations of rubberlike solids can be described by models such as Polynomial, Ogden, Mooney-Rivlin, neo-Hookean, reduced polynomial and Yeoh strain energy functions. All these models allow the material coefficients to be determined directly from experimental test data. The Yeoh polynomial model was choosen because it is often used to describe elastic behaviour over large ranges of strain with reasonable success [11].

\section{DESIGN IMPLEMENTATION}

\section{A. Fabrication of Soft Actuators}

The silicone based soft robots was produced by a 2-part moulding process. Designing the actuator geometry of the mould is therefore required before moulding can commence. A 3D CAD design program, SolidWorks was used to design the geometry. For one soft robot, two moulds are required a top mould and a bottom mould. The top mould constitute the actuator geometry while the bottom mould is a flat base layer as shown in Figure 2. The moulds are printed out with a HP Designjet(R) 3D printer using Acrylonitrile Butadiene Styrene (ABS) plastic material.

Soft actuators for bending motion were manufactured using two grades of silicon highly extensible and high stiffness silicone. Ecoflex 0030 with a viscosity of $3000 \mathrm{cP}$ was used as the soft silicone while Addition Cure 33 Silicone Rubber with a viscosity of $7000 \mathrm{cP}$ was used as the hard silicon. Each of the silicone grades come in two parts Parts $\mathrm{A}$ and $\mathrm{B}$, to achieve moulding, both parts of the silicon grade are mixed in equal quantity (by weight or volume), poured into the 3D printed mould and allowed to cure at room temperature for 4 hours. In order to accomplish a good glue between the bottom (hard) and top (soft) layer, the top layer is first moulded and cured, it is then removed from the mould and allowed to sit on the bottom layer of liquid silicon. This 


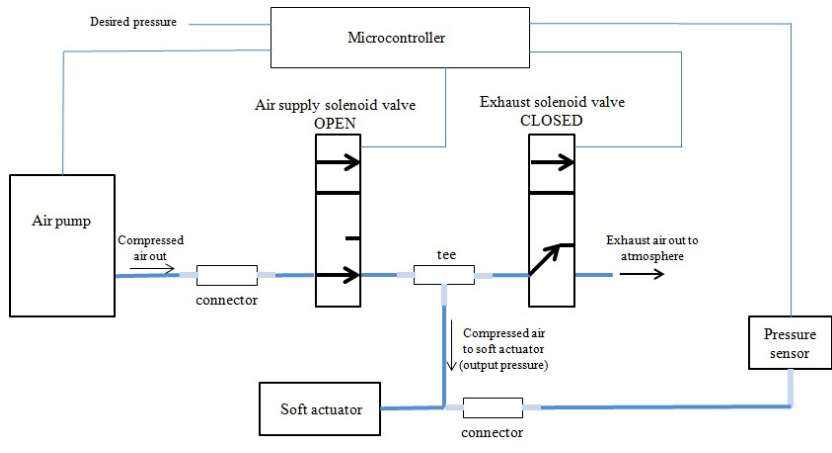

Figure 3. Circuit arrangement of soft robot pressure controller

ensured perfect seal because cured silicone bonds well with liquid silicon and the result produces an ideal continuous structure. A silicone tube is then inserted to a create a air passage from a pressure source to the designed soft robot. This is done by creating a hole on the body of the soft robot into the air channel. After insertion of the tubing, additional liquid silicone is used to seal the ends of the hole created in order to minimize air leakage. To facilitate easy removal of the cured silicone from the mould, clear acrylic lacquer or petroleum jelly is applied to the mould surface prior to pouring the liquid silicone in the mould.

The principle used to achieve bending motion is the same as that of a bimetallic strip, that is, two materials of different expansion coefficient or young modulus glued together will bend when heated. For the case of the soft robot, the high stiffness silicon is used as the bottom layer while the low stiffness but highly extensible silicon is used as the top layer which has the air channel. To produce bending, compressed air from a pressure source is fed into the soft robot via air tubing. The soft robot is then able to bend because of the difference in young modulus of the bottom and top silicone.

\section{B. Pressure Regulator Circuit}

Figure 3 shows the arrangement of pumps and valves for the actuation of soft robots in order to carry out inflation and deflation cycles. As shown, it consists of an air pump to act as the pressure source; two solenoid valves (one acts as an air supply valve while the other as an exhaust valve) and a pressure sensor to measure the air pressure in the soft actuator.

Compressed air from the pump passes through the air supply solenoid valve and changes to output pressure when the air supply solenoid valve turns ON. In this way, air from the supply pump passes through the air supply solenoid valve and changes to output pressure. A PWM output is then produced on the output pin of the MCU to switch ON/OFF the exhaust valve in order to produce an output pressure equal to the desired pressure. The exhaust valve is also used to deflate the soft actuator - this is essential for crawling [2] or snake like [3] motion of soft robots. The output pressure

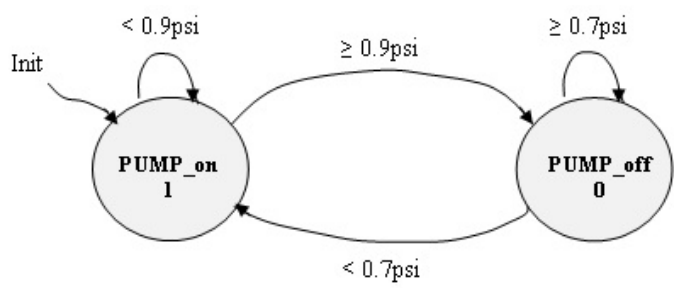

Figure 4. FSM to control pressure inside soft robot

is fed back to the MCU via the pressure sensor. This is to check if the desired pressure has become equal to the output pressure. Pressure corrections then occur to produce an output pressure that is equal to the set pressure. Once the pressure sensor has sensed that the desired pressure is equal to the output pressure, the exhaust valve will turn OFF (close) in order to maintain a constant pressure.

To maintain the air pressure inside the soft robot, a Finite State Machine (FSM) (Figure 4) can be used. For example, to control the air pressure between 0.7 psi to $0.9 \mathrm{psi}$, two pressure thresholds are used to prevent the pump from toggling on/off/on/off/on... too quickly. If the system were to be in the PUMP-off state (speed of air pump is reduced), then the system would remain in the PUMP-off state until the pressure drops below $0.7 \mathrm{psi}$. If the system were to be in the PUMP-on state (air pump is inflating the robot at maximum duty cycle), then the system would remain in the PUMP-on state until the pressure rises above $0.9 \mathrm{psi}$.

More than one separate individual units of a soft actuator can be controlled using this system. This would be achieved by the addition of two valves for every separate air channel. This is a low pressure controller system that operates at about 0 to $5 \mathrm{psi}$; since the pressure range of the pump and solenoid valves are between 0 - $6 \mathrm{psi}$. Soft actuators operate within this pressure range and so this system is suitable for actuating soft robots. To achieve a high pressure system, this pressure regulator circuit is easy to scale up by using a high pressure pump and solenoid valves.

\section{Production of $P C B$}

The pressure regulator circuit was implemented on a printed circuit board as shown in Figure 5 to achieve a compact and portable system. The components used include the following:

1) Microcontroller: The PIC16F876A microcontroller is the brain of the regulator system. Here, control commands are written to inflate/deflate the robot, read the system pressure from the pressure sensor to be used as an appropriate feedback and write the current pressure on the LCD display.

2) Yellow LED: The LED was used as an indicator for the system.

3) LCD: A LM016 16-character, 2-line LCD was used to display the output pressure. It acts as an indicator for the user of this embedded system. 


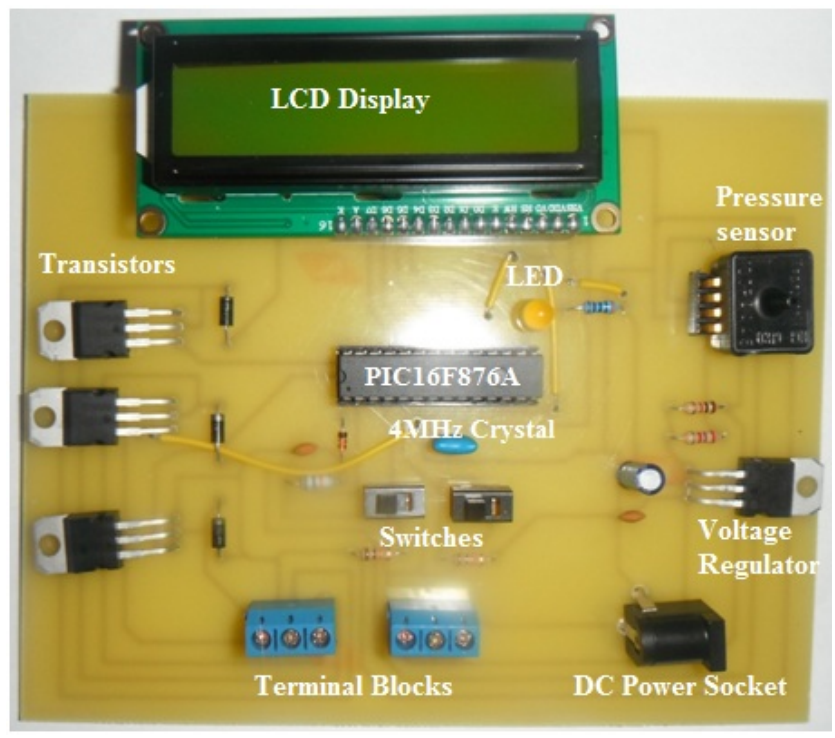

Figure 5. Produced Printed Circuit Board

4) ON/OFF Switches: When Switch 1 is turned on the indicating LED would come ON. Switch 2 is used to turn on/off the valves and pump so that the user has control of the start and stop operation of the system.

5) Pressure Sensor: The ASDX analogue pressure sensor was required to monitor the pressure and provide an appropriate feedback for the regulator system.

6) Transistors: TIP120 Darlington transistors were used to interface the high current external devices to the microcontroller. The pump and solenoid valves make use of high current and cannot be directly connected to the digital I/O pins of the microcontroller. Transistors were used for the solenoid valves and pump to drive them at their appropriate current while ensuring that they are controlled by the MCU. When the output pin of the MCU to which the valve/pump is connected to is high $(5 \mathrm{~V})$, the transistor is active, when the output pin is low $(0 \mathrm{~V})$, the transistor is off. In general, the transistor is used to switch current on and off to the solenoid valves and pump; through the transistor, a small current supplied by the microcontroller will switch on a large current that drives the valves and pump.

7) Diodes: The 1N4001 diodes were used as flyback diodes for the valves and pump. When the pump or valve is subjected to a large change in current, such as when the transistor switches, the inductor in the pump and valves presents a large back-emf (back-electromotive force or voltage). This large voltage spike could be harmful, so flyback diodes were required to dissipate this spike.

8) Power Supply: This regulator system was powered by a 6V DC power adapter plugged to a socket outlet.

9) Voltage Regulator: The voltage regulator was required to produce a $5 \mathrm{~V}$ DC output from the $6 \mathrm{~V}$ DC input in order to power up the PIC microcontroller at the appropriate voltage.

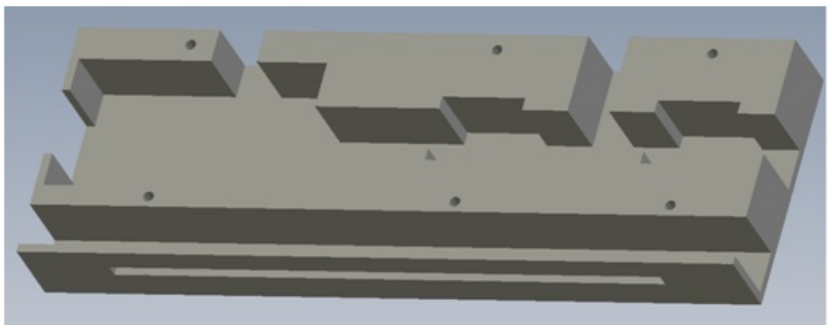

Figure 6. 3D casing for valves and pump in SolidWorks

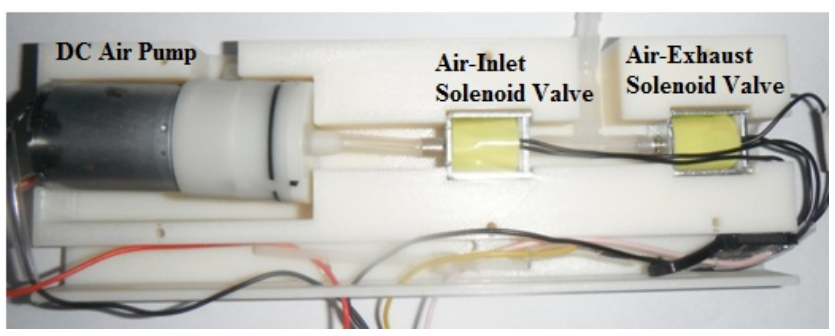

Figure 7. 3D Printed casing housing the valves and pump

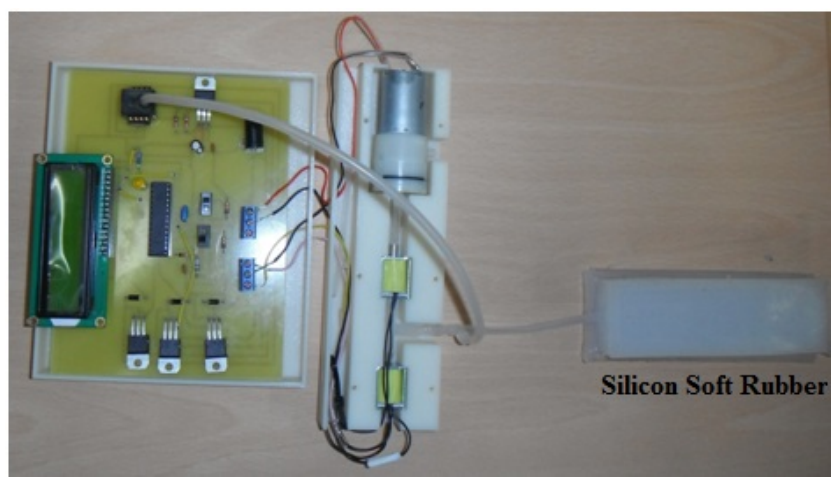

Figure 8. The complete setup for soft actuation

10) Terminal Blocks: They were used to connect the wires from the valves and pump to the circuit board layout as shown in Figures 5 and 7.

\section{Production of 3D-Printed Casing}

A 3D casing for the valves and pump was designed using SolidWorks 3D CAD design software (Figure 6). This was done to act as housing for the pump and solenoid valves so as to achieve a fully embedded and compact system. The 3D model was printed with ABS plastic using a 3D printer. Figure 7 shows the solenoid valves and pump inside the 3D-printed casing.

The completed hardware system including a one-channel soft robot is shown in Figure 8. A silicone tubing is connected from the pressure sensor to the inlet of the silicone soft actuator as shown to measure the air pressure therein. 


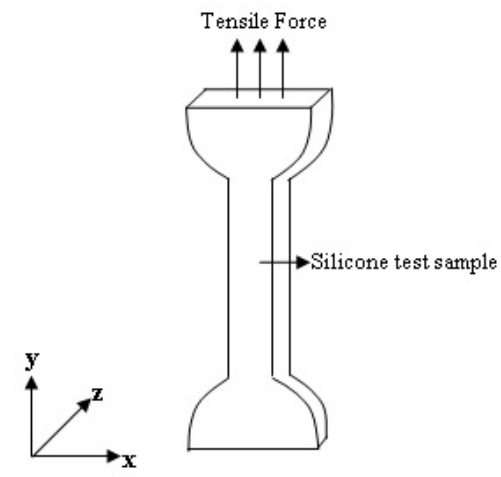

Figure 9. Uniaxial Tensile Test

\section{E. Uniaxial Tensile Test}

Uniaxial tensile test (Figure 9) of Ecoflex 0030 and Addition Cure Tiranti 33 test samples were carried out at room temperature by applying a predefined tensile force and measuring the elongation. Instron tensile testing machine was used at a rate of $30 \mathrm{~mm} / \mathrm{min}$. Because silicone based soft robots are hyperelastic in nature, their large strain elastic response is modelled using the strain energy potential [11]. The nominal stress and strain of the tensile data was plotted and the polynomial function of Yeoh was used to fit the data using a general purpose Finite Element program, ABAQUS. The Yeoh model is one of the forms of the polynomial model of strain energy potential. As observed from Figures 10 and 11, the polynomial form provided a good fit to the experimental data. This is because the strain from experimental data remained within the strain magnitude where the data and the hyperelastic model fit well as shown. However, the computational and experimental results for the uniaxial tension tests match well at strains less than $100 \%$, further work will include obtaining sufficient material data points at strains less than $100 \%$ and performing Finite Element Analysis (FEA) to compare experimental results with simulated results.

\section{Performance Evaluation}

The PIC16F876A switches its output pins between 5V and $0 \mathrm{~V}$ to create a PWM signal for controlling the speed of the air pump. PWM works because the motor in the air pump has rotational inertia that filters out the fast switching. Therefore, the rotational inertia of the DC motor inside the pump smoothens out these rapid pulses provided by PWM and the result is a slower axle rotation and consequently regulating air pressure. For the case of the solenoid valves, PWM produced switching noise. This can be prevented by switching the valves either ON or OFF for inflation and deflation cycles while using PWM to control the speed of the air pump and therefore control pressure of air in the soft module.

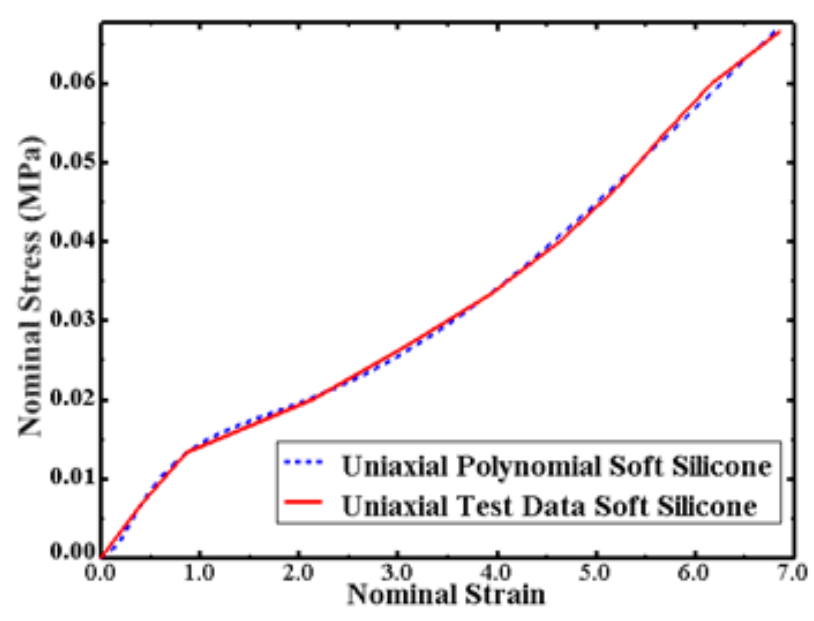

Figure 10. Strain curves of Ecoflex 0030 Silicone (Experimental and Polynomial model data)

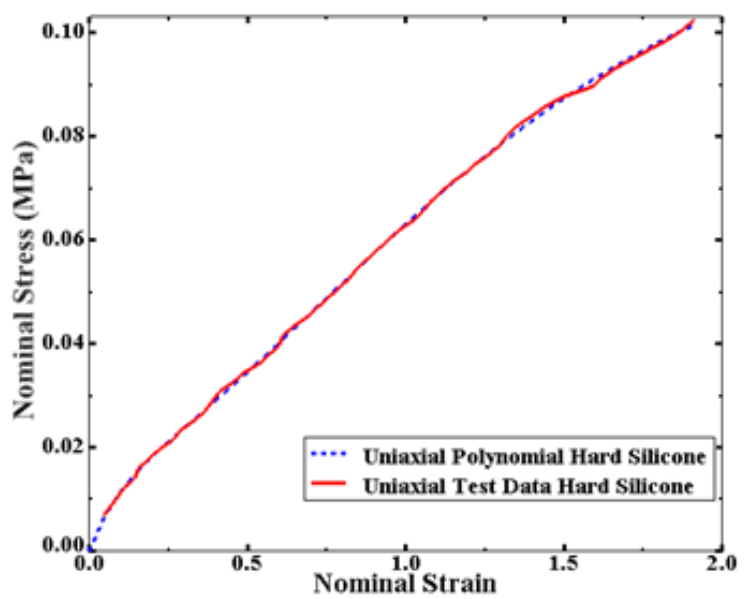

Figure 11. Strain curves of Addition Cure 33 Silicone (Experimental and Polynomial model data)

The PIC16F876A microcontroller ADC converts voltage into a 10-bit digital number (0 to 1023). To acquire voltage readings from the pressure sensor using the microcontroller based control system, it is important to maintain an accurate sampling rate for the purpose of reading data from the pressure sensor and outputting commands to the actuators. The time in between ADC samples must be equal and known in order for the digital signal processing to function properly. The most accurate sampling method is timer-triggered sampling. Timer2 periodic interrupt on the PIC16F876A was used, the precision is 8 bits and the resolution is a bus cycle time of $0.25 \mu \mathrm{s}$. The ADC was configured to sample a single channel at a periodic rate of $250 \mathrm{~Hz}$. Inter-thread communication was done to write the updated value of the pressure readings to the LCD using a binary semaphore flag.

Results shows the suitability and performance of this elec- 


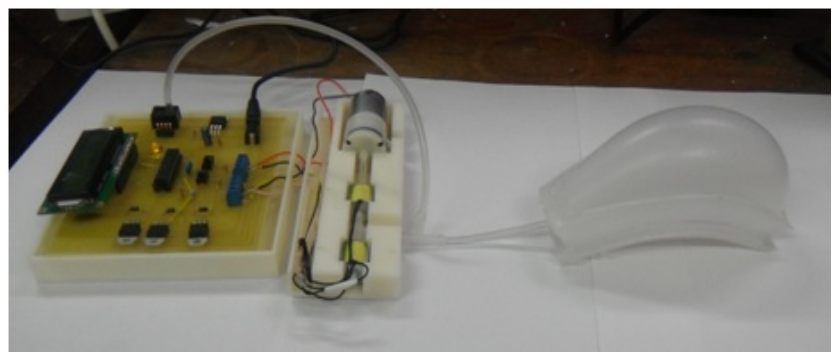

Figure 12. Using the completed system to actuate a silicon soft robot

tronic hardware system when actuating soft robots operating at low pressures $(<5 \mathrm{psi})$. Figure 12 shows the completed system in operation as it actuates a soft robot to produce bending motion. Results obtained from the pressure sensor (Figure 13) shows the pressure of air in the soft module displayed on the LCD. This provides a good measure of the pressurized air in the soft actuator. It can be noticed from Figure 13 that the two least significant digits on the LCD flickered, this flickering is caused by electrical noise in the circuit. Figure 14 shows the performance of the designed hardware for different designs of soft pneumatic actuators.

The total cost for the whole system including cost of valves, pump, silicon tubes, connectors, pressure sensor and electronic components is within a manageable budget and therefore cost of hardware production is low. In general, the more ambitious the requirements for control are, the higher the costs will be while the system compactness reduces. The higher the required pressure, the larger and costly the solenoid valves will be. The size of the valves is in contradiction to compactness requirements. This also applies to the time taken for the control program to run given the clock speed of the MCU. The extent of compactness is reliant on the degree of integration of control components such as sensors, valves and control logic. For a 2 or more actuator system such as a 5-legged crawling soft robot, the number of transistors, pressure sensor and solenoid valves increases accordingly. The compactness also depends on the amount of tubing and cables needed for operation.

\section{RESULTS}

When silicon soft actuators are pressurized, the channel enclosed by soft silicone undergoes high strain and lengthens while the base stiffer layer acts as a constraint, therefore, the expansion generates a bending curvature about the stiffer but extensible layer. To determine the degree of bending that this electronic system can achieve for the one-channel soft robot in Figure 8, a graph of the bending distance as a function of pressure was plotted (Figure 10). The bending distance is defined by the distance between the mid-point of the bottom layer of the soft robot and the ground support in its uninflated state and the distance of this mid-point from the ground support when pressurized.

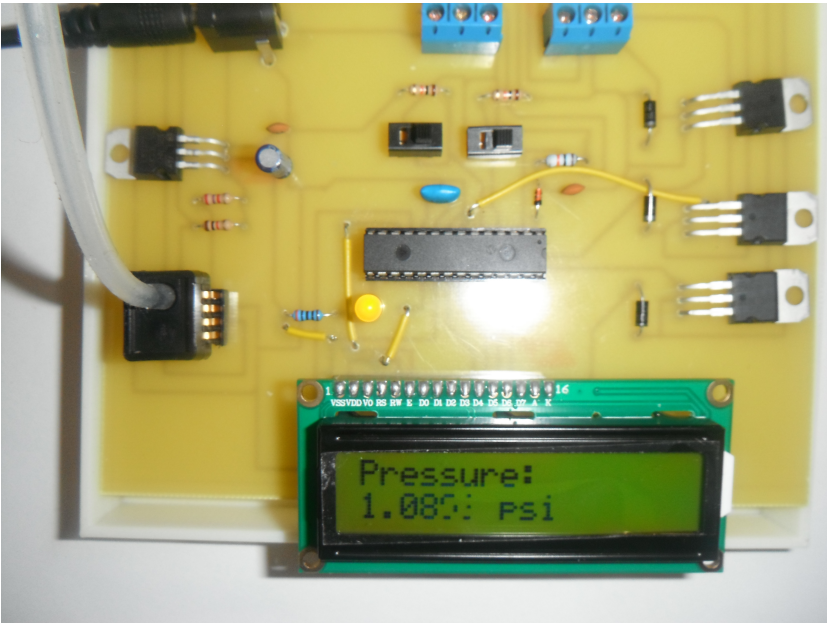

Figure 13. LCD displaying the pressure of air inside the soft actuator

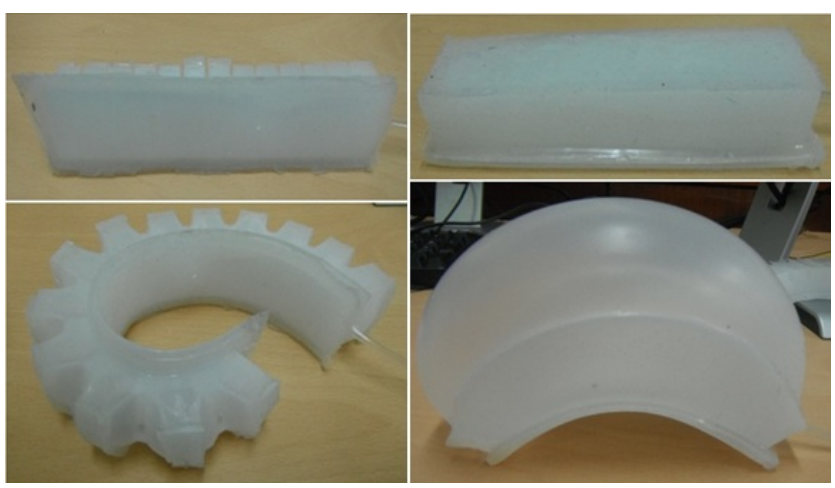

Figure 14. Control hardware used to actuate different designs of soft actuators (before and after inflation)

To determine this distance, visual processing was performed by using a Webcam to determine the position of three colour markers (Figure 16). The visual processing program was written in $\mathrm{C}++$ programming language with the use of OpenCV libraries. A displacement of $6 \mathrm{~mm}$ was measured as the distance moved by this soft actuator at the maximum bending. Figure 17 shows that the time taken to inflate the soft robot to its maximum bending is about 10 s for the inflation cycle while the deflation time as shown in Figure 18 is about 8s. Factors such as exhaust speed of the valves, power rating of the pump, maximum pressure of valves and pump, length of silicone tubes will affect the time duration of inflation and deflation cycles.

Using the characteristic graph of pressure and radius of curvature of the bending soft actuator to obtain interpolation data, the MCU can then be programmed to approximately set the curvature given a set pressure. For this system, it can be difficult to achieve high accuracy of pressure and curvature measurements. This is due to the accuracy of the control and also due to some parameters of the 


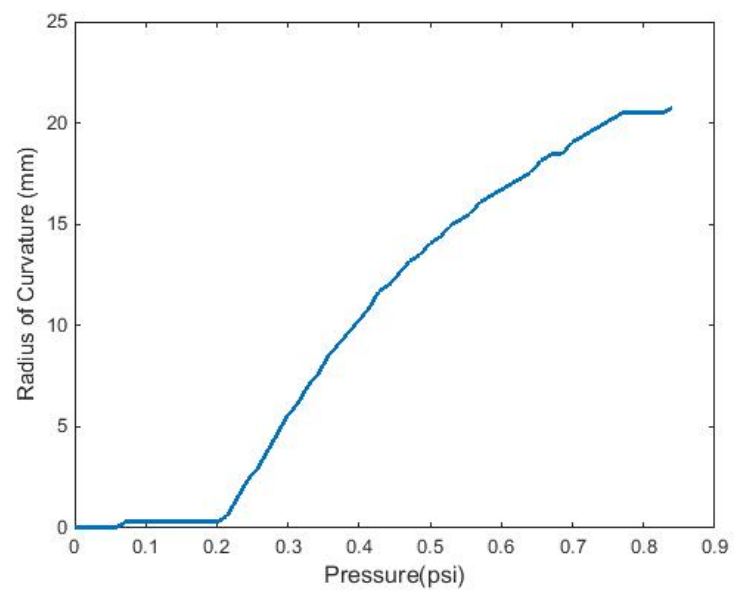

Figure 15. Bending distance as a function of pressure

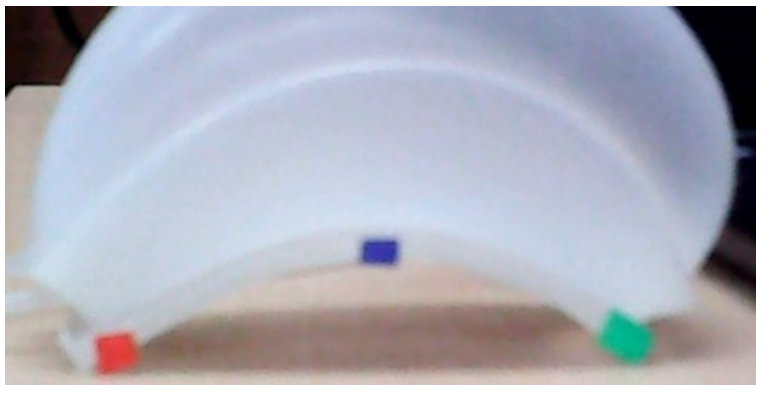

Figure 16. Colour markers used to measure the radius of curvature

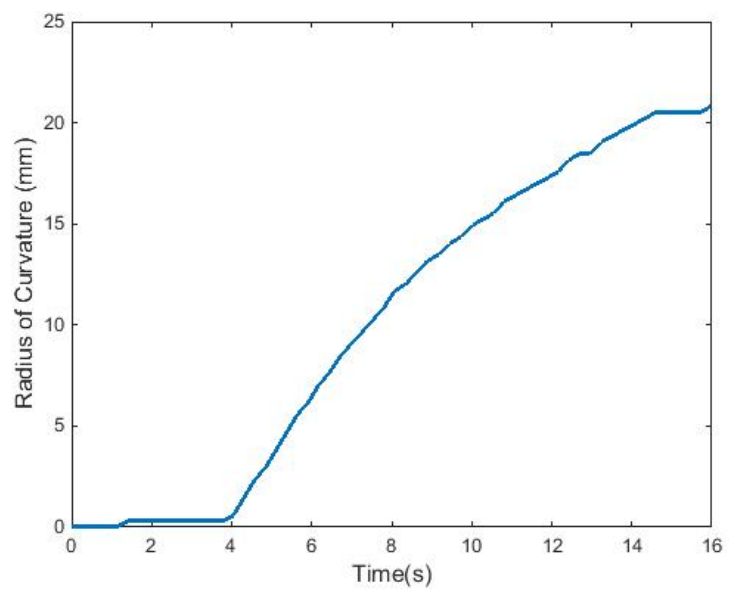

Figure 17. Bending distance as a function of time (inflation cycle)

solenoid valve such as leakage and exhaust speed as well as limited curvature measurement accuracy through the use of visual information from a camera. Also, air leakage at the connecting point between the soft robot and the tubing; loose tubing connection between the pump, valves and pressure sensor will limit the accuracy of this system. Using a 16-

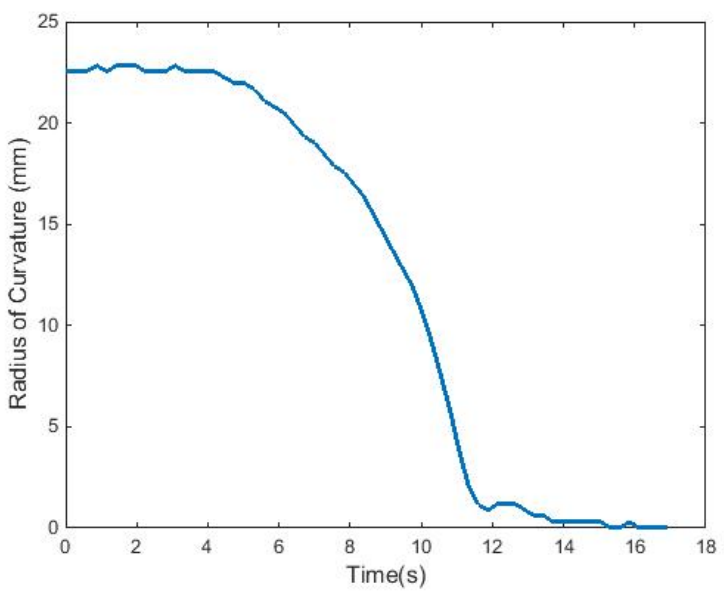

Figure 18. Bending distance as a function of time (Deflation cycle)

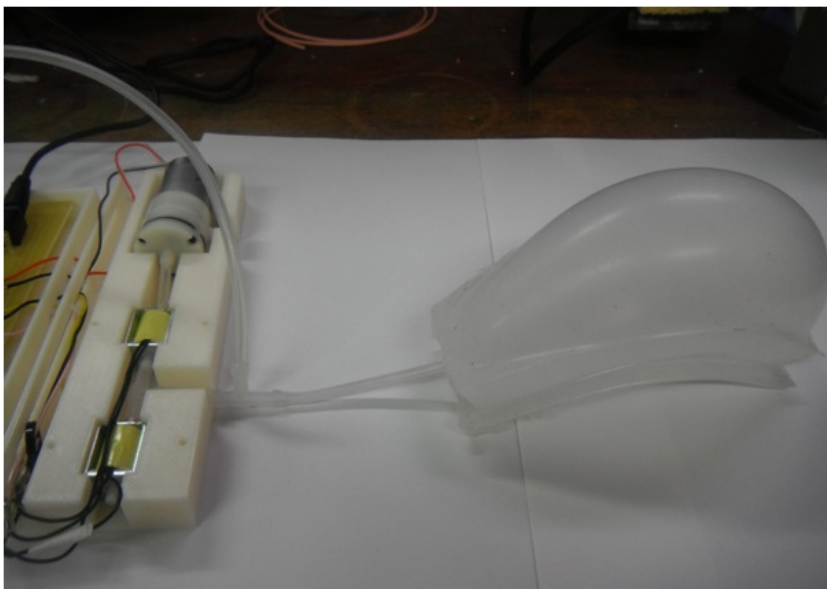

Figure 19. Completed system in operation

bit ADC to collect pressure data will improve accuracy of pressure measurements. Likewise, an MCU running at a higher clock frequency can be used so that tuning and improved quality of embedded control is realized.

\section{CONCLUSION}

The design of an MCU controlled and operated pressure regulator system to perform pneumatic actuation of soft robots was presented. An approach to using this embedded system to actuate a single air channel soft robot was demonstrated. The challenges regarding production of a compact electronic circuit for soft robots have been addressed. This embedded hardware approach is very promising and can be easily modified to act as a pressure regulator for water by simply replacing the air pump with a water pump and providing suitable valves.

The main limitation of this system is that it can only be used for inflating one continuous air channel. Nevertheless, the system can be easily modified to allow for two, three 
or more actuators or for McKibbean muscles whereby each muscle will be actuated by 2 solenoid valves. This will also be required in cases requiring 2-joint control necessary for elbow motion or multi-finger actuator where each of the fingers requires a pair of solenoid valves for grasping tasks. For high pressure systems, the air source may be excluded from the board so as to reduce the size and weight, using this approach will allow for the use of an air tank containing compressed air as the pressure source.

The system is modular in terms of mechanical modularity, supply of compressed air, electrical power supply and system integration as all of these are on the PCB board and held in a casing. The control algorithms of this system are relatively simple, it is therefore very easy to modify the computer program to cover a wide range of movements such as crawling, bending, etc required for soft robots. Future research work will include perforimg FEA to compare experimental results of bending motion of the soft robots with model data. Also, more work will include using this hardware system to actuate soft robots designed for neuro-rehabilitation. This design will also be extended to facilitate its use for complex motions as well as for actuating soft robots having more than one pneumatic network.

\section{ACKNOWLEDGMENT}

The authors would like to thank Prof. William Harwin for fruitful discussions, Dr. Guy Haworth for his support, Mr. Ben Haworth for his master's thesis, Mr. Steve Gould and Mr. Nick Dove for their help in producing the circuit board and The Great Britain Sasakawa Foundation (Project Number: 4391) for the international exchange program.

\section{REFERENCES}

[1] F. Ilievski, A. D. Mazzeo, R. F. Shepherd, X. Chen, and G.M. Whitesides, Soft Robotics for Chemists, Angewandte Chemie, Vol. 123, pp. 1930 - 1935, 2011.

[2] R. F. Shepherd, F. Ilievskia, W. Choia, S. A. Morina, A. A. Stokes, A. D. Mazzeoa, X. Chena, M. Wanga and G. M. Whitesides, Multi-gait soft robot, PNAS, 2011.

[3] A. D. Marchese, R. K. Katzschmann and D. Ruis, Whole Arm Planning for a Soft and Highly Compliant 2D Robotic Manipulator, IEEE International Conference on Intelligent Robots and Systems, pp 554 - 560, 2014.

[4] P. Maeder-York, T. Clites, E. Boggs, R. Neff, P. Polygerinos, D. Holland, L. Stirling, K. C. Galloway, C. Wee, C. J. Walsh, Biologically Inspired Soft Robot for Thumb Rehabilitation, Proceedings of Design of Medical Devices Conference, 2014.

[5] R. F. Shepherd, A. A. Stokes, F. Freake, J. Barber, P. W. Snyder, A. D. Mazzeo, L. Cademartiri, S. A. Morin, and G. M. Whitesides, Using explosions to power a soft robot, Angewandte Chemie International Edition, pp 2892 - 2896, 2013.

[6] N. Napp, B. Araki, M. T. Tolley, R. Nagpal, and R. J. Wood, Simple passive valves for addressable pneumatic actuation, IEEE International Conference on Robotics and Automation, pp 1440 - 1445, 2014.

[7] E. Matter, Biomimetic Dexterous Hands: Human Like Multi-fingered Robotics Hand Control, International Conference on Modelling and Simulation, pp 193 - 200, 2012.

[8] M. Mihajlov, M. Hubner, O. Ivlev and A. Graser, Modelling and control of fluidic robotic joints with natural compliance, IEEE International Conference on Control Applications Computer Aided Control System Design, pp. 2498 - 2503, 2006.
[9] M. Jordan, D. Pietrusky, M. Mihajlov, and O. Ivlev, Precise position and trajectory control of pneumatic soft-actuators for assistance robots and motion therapy devices, IEEE International Conference on Rehabilitation Robotics, pp. 663 - 668, 2009.

[10] A. Wilkening, M. Mihajlov, and O. Ivlev, Model-based pressure and torque control for innovative pneumatic soft-actuators, Proceedings of International Confeence on Fluid Power, Vol. 4, Aachen, Germany, pp. $291-302,2010$.

[11] A. N. Gent, M. D. Ellul, R. H. Finney, G. R. Hamed, D. L. Hertz, F. O. James, G. D. Lake, T. S. Miller and R.P. Campion, Engineering with Rubber: How to Design Rubber Components, Carl Hanser Verlag GmbH \& Co. KG, 3rd Edition, 2012. 